\title{
Simple Generalized Group-Oriented Cryptosystems Using ElGamal Cryptosystem
}

\author{
Chou-Chen YANG, Ting-Yi CHANG, Jian-Wei LI, \\ Department and Graduate Institute of Computer Science and Information Engineering \\ Chaoyang University of Technology \\ 168, Gifeng E. Rd., Wufeng, Taichung County, Taiwan 413, R.O.C. \\ e-mail: ccyang@mail.cyut.edu.tw
}

\author{
Min-Shiang HWANG \\ Department of Information Management \\ Chaoyang University of Technology \\ 168, Gifeng E. Rd., Wufeng, Taichung County, Taiwan 413, R.O.C. \\ e-mail: mshwang@mail.cyut.edu.tw
}

\section{Received: December 2002}

\begin{abstract}
In the generalized group-oriented cryptosystem, the sender can send a conditional message to a group of users such that only the specified sets of users in this group can cooperate to decrypt this message. In this paper, we will use an ElGamal cryptosystem and an elliptic curve ElGamal cryptosystem to achieve the purposes of generalization and group-orientation, respectively. Both of our schemes are more efficient than Tsai et al.'s scheme in terms of sender's computational complexity.
\end{abstract}

Key words: Diffie-Hellman scheme, ElGamal cryptosystem, elliptic curve cryptosystem, grouporiented cryptosystem.

\section{Introduction}

The concept of group-oriented cryptosystem was first introduced by Desmedt (1987). In the generalized group-oriented cryptosystem (GGOC), a sender sends an encrypted message to a group such that the received message can only be decrypted by the authorized subsets of users in the receiver group. An authorized subset of the receiver group is called an access instance denoted as $f$. The collection of the access instances for a particular type of message is called the access structure denoted as $F$. An access structure can be denoted in the disjunctive normal form (DNF), i.e., $F=f_{1}+f_{2}+\cdots+f_{k}$. Let $U_{1}, U_{2}, \cdots, U_{N}$ be all of the users in the group, and the access instances $f_{1}=U_{1} U_{2} U_{4}$, $f_{2}=U_{3} U_{5}, f_{3}=U_{7}$. The access structure can be represented as $F=U_{1} U_{2} U_{4}+U_{3} U_{5}+$ $U_{7}$. The ciphertext sent by a sender can only be decrypted by the cooperation of either $U_{1}, U_{2}$ and $U_{4}$ or $U_{3}$ and $U_{5}$ or $U_{7}$ alone. If $F=U_{1}+U_{2}+\cdots+U_{N}$, it means the urgent message can be decrypted by any user in the group. 
Lin and Chang (1994) proposed a GGOC based on the Diffie-Hellman key distribution scheme (Diffie and Hellman, 1976). Later, Tsai et al. (1999) proposed a more efficient GGOC than Lin and Chang's scheme that was also based on the Diffie-Hellman key distribution scheme. However, Lin and Chang's scheme and Tsai et al.'s scheme employed none of the existing asymmetric cryptosystems (ElGamal, 1985; Hwang et al., 2002). They proposed new schemes to live up to the requirements of GGOC and used additional asymmetric cryptosystems to encrypt/decrypt the message. The computational complexity on the sender's side increases, causing both the number of users in the access structure and that of the access instances to grow in (Lin and Chang, 1994) and making the number of users in the access structure in (Tsai et al., 1999) go up.

In this paper, we will propose a new GGOC to further reduce the computational complexity on the sender's side, and there will be no symmetric cryptosystem to encrypt/decrypt the message. Our scheme only uses the ElGamal cryptosystem (ElGamal, 1985) to achieve the purpose of generalization and group-orientation. Compared with Tsai et al.'s scheme, our scheme performs better in lowering the computational complexity of the sender. On the other hand, many researchers have explored the concept of elliptic curve cryptosystem, which was firstly proposed by Miller (1986) and Koblitz (1987). The elliptic curve cryptosystem provides smaller key sizes, more bandwidth savings and faster implementations, features which are especially attractive to applications of security demands (Koblitz et al., 2000). Taking advantage of such features, we will also propose the elliptic curve version of our proposed GGOC.

This article is organized as follows: In Section 2, we will briefly review Tsai et al.'s GGOC. In Section 3, our new GGOC scheme based on ElGamal cryptosystem will be proposed, and the security will be analyzed. Moreover, the elliptic curve version of our proposed GGOC will also be presented. In Section 4, the performance of our schemes and Tsai et al.'s scheme will be compared. In Section 5, there will be some discussions. Finally, Section 6 will present our conclusion.

\section{Review of Tsai $e t$ al.'s GGOC}

In this section, we shall briefly review Tsai et al.'s scheme. Assume that $U_{0}$ is the sender, and $U_{1}, U_{2}, \cdots, U_{N}$ are all the users in the receiver group. Let $p$ be a large prime such that $p-1$ has a large prime factor with order $q$ in the Galosis field $G F(p)$. Each user $U_{i}$ in the group has a secret key $x_{i}$ in $G F(p)$ and the corresponding public key $y_{i}=g^{x_{i}} \bmod p$, for $i=1,2, \cdots, N$. Anyone can get the public keys via some authentication service (e.g., the X.509 directory authentication service (Stallings, 1999)). To send the message $M$ to the group, the sender $U_{0}$ firstly determines the access structure $F=f_{1}+f_{2}+\cdots+f_{k}$ for $M$. Assume that $U_{1}, U_{2}, \cdots, U_{n}(n<=N)$ are all the users in the access structure. Then $U_{0}$ performs the following steps.

Step 1. Choose a random number $r$ in $G F(p)$ and compute $b=g^{r} \bmod p$.

Step 2. Compute $t_{i}=\left(y_{i}\right)^{r} \bmod p$, for $i=1,2, \cdots, n$. 
Step 3. Choose a random encrypting key $K e y$ in $G F(p)$ and compute the ciphertext $C=E_{K e y}(M)$, where $E$ is the encryption algorithm in the symmetric cryptosystem such as DES (Smid and Branstad, 1988) and Rijndael (Daemen and Rijem, 1999; Daemen and Rijem, 2001).

Step 4. Compute $r_{j}=K e y \oplus\left(\prod_{U_{i} \in f_{j}} t_{i} \bmod p\right)$, for $j=1,2, \cdots, k$.

Step 5. Send $\left\{F, b, r_{1}, r_{2}, \cdots, r_{k}, C\right\}$ to the receiver group.

After receiving $\left\{F, b, r_{1}, r_{2}, \cdots, r_{k}, C\right\}$, the users $U_{i} \mathrm{~s}$ (for $i=j_{1}, j_{2}, \cdots, j_{v}$ ) in the access instance $f_{j}$ can cooperate to decrypt the message by using their secret keys as follows:

Step 1. Compute $t_{i}=b^{x_{i}} \bmod p$, for $i=j_{1}, j_{2}, \cdots, j_{v}$.

Step 2. Compute Key $=r_{j} \oplus\left(\prod_{U_{i} \in f_{j}} t_{i} \bmod p\right)$.

Step 3. Recover $M=D_{K e y}(C)$, where $D$ is the encryption algorithm in the symmetric cryptosystem.

In Tsai et al.'s scheme, each user $U_{i}$ in the receiver group has the secret key $x_{i}$ and the corresponding public key $y_{i}$ in the system. However, to live up to the requirements of GGOC, their scheme requires an additional symmetric cryptosystem to encrypt/decrypt the message.

\section{Our Proposed GGOC Schemes}

In this section, we will first propose our GGOC scheme with the ElGamal cryptosystem and then present the elliptic curve version of our proposed scheme.

\subsection{GGOC with the ElGamal Cryptosystem}

The parameters $\left(p, q, g, x_{i}, y_{i}\right)$ are the same as those in Tsai et al.'s scheme. Suppose $U_{0}$ wants to send the message $M$ to the access structure $F=f_{1}+f_{2}+\cdots+f_{k}$, and $U_{1}, U_{2}, \cdots, U_{n}$ are all the users in the access structure. Then $U_{0}$ performs the following steps.

Step 1. Choose a random number $r$ in $G F(p)$ and compute $b=g^{r} \bmod p$.

Step 2. Compute $C_{j}=M \cdot\left(\prod_{U_{i} \in f_{j}} y_{i}\right)^{r} \bmod p$, for $j=1,2, \cdots, k$.

Step 3. Send $\left\{F, b, C_{1}, C_{2}, \cdots, C_{k}\right\}$ to the receiver group.

After receiving $\left\{F, b, C_{1}, C_{2}, \cdots, C_{k}\right\}$, the users $U_{i} \mathrm{~s}$ (for $i=j_{1}, j_{2}, \cdots, j_{v}$ ) in the access instance $f_{j}$ can cooperate to decrypt the message by using their secret keys as follows:

Step 1. Compute $t_{i}=b^{x_{i}} \bmod p$, for $i=j_{1}, j_{2}, \cdots, j_{v}$.

Step 2. Recover $M=C_{j} \cdot\left(\prod_{U_{i} \in f_{j}} t_{i}\right)^{-1} \bmod p$. 
In our scheme, the sender encrypts the message by multiplying the users' public keys $y_{i}$ s to be the public key in the original ElGamal cryptosystem. Then, the users $U_{i}$ s in the access instance $f_{j}$ can cooperate to recover the message $M$.

Theorem 1. The users $U_{i}$ s (for $i=j_{1}, j_{2}, \cdots, j_{v}$ ) in the access instance $f_{j}$ can cooperate to recover the message $M$.

Proof. Because

$$
\begin{aligned}
C_{j} & =M \cdot\left(\prod_{U_{i} \in f_{j}} y_{i}\right)^{r} \bmod p \\
& =M \cdot\left(\prod_{U_{i} \in f_{j}} g^{x_{i}}\right)^{r} \bmod p \\
& =M \cdot\left(g^{\sum_{U_{i} \in f_{j}} x_{i}}\right)^{r} \bmod p,
\end{aligned}
$$

thus

$$
\begin{aligned}
M & =C_{j} \cdot\left(g^{\sum_{U_{i} \in f_{j}} x_{i}}\right)^{-r} \bmod p \\
& =C_{j} \cdot\left(g^{\sum_{U_{i} \in f_{j}} x_{i} \cdot r}\right)^{-1} \bmod p \\
& =C_{j} \cdot\left(\prod_{U_{i} \in f_{j}} t_{i}\right)^{-1} \bmod p .
\end{aligned}
$$

According to the descriptions above, in our scheme, if the access structure has only one single user and the sender encrypts the message for each user in this access structure, then the function of our scheme is exactly the same as that of the ElGamal cryptosystem.

It is clear that the security of the proposed GGOC is based on the ElGamal cryptosystem, which in turn is based on the intractability of the discrete logarithm problem (DLP). It is very difficult for an adversary to compute the secret key $x_{i}$ of user $U_{i}$ from the equation $y_{i}=g^{x_{i}} \bmod p$. For the same reason, to obtain the random number $r$ generated by $U_{0}$ from the equation $b=g^{r} \bmod p$ is also difficult. In addition, it is also very difficult for the legal users $U_{i} \mathrm{~s}$ (for $i=j_{1}, j_{2}, \cdots, j_{v}$ ) in the access instance $f_{j}$ to reveal secret keys $x_{k} \mathrm{~s}$ of other users $U_{k} \mathrm{~s}$ from the equation $t_{k}=b^{x_{k}} \bmod p\left(i \neq k, k=j_{1}, j_{2}, \cdots, j_{v}\right)$. On the other hand, to recover $M$ from the message $\left\{F, b, C_{1}, C_{2}, \cdots, C_{k}\right\}$ sent by $U_{0}$, the adversary has to break the Diffie-Hellman scheme and find all the terms $t_{i}$ s without knowing $x_{i} \mathrm{~s}, i \in f_{j}$.

\subsection{GGOC with the Elliptic Curve ElGamal Cryptosystem}

Let $E$ be the elliptic curve defined over a finite field $F_{2^{m}}$, and let $G$ be a publicly known base point with order $p$ on $E$, preferably a generator of $E$ (Koblitz et al., 2000). Each user $U_{i}$ in the group has a secret key $x_{i} \in[1, p-1]$ and the corresponding public key $Y_{i}=x_{i} \cdot G$, for $i=1,2, \cdots, N$. Similarly, suppose $U_{0}$ wants to send the message $M$ to 
the access structure $F=f_{1}+f_{2}+\cdots+f_{k}$, and $U_{1}, U_{2}, \cdots, U_{n}$ are all the users in the access structure. Then $U_{0}$ performs the following steps.

Step 1. Choose a random number $r \in[1, p-1]$ and compute the point $B=r \cdot G$.

Step 2. Express $M$ as the $x$-coordinate of a point $P_{M}$ on $E$ (Koblitz, 1998). Then, compute $C_{j}=P_{M}+\left(\sum_{U_{i} \in f_{j}} Y_{i}\right) \cdot r$, for $j=1,2, \cdots, k$.

Step 3. Send $\left\{F, B, C_{1}, C_{2}, \cdots, C_{k}\right\}$ to the receiver group.

After receiving $\left\{F, B, C_{1}, C_{2}, \cdots, C_{k}\right\}$, the users $U_{i} \mathrm{~s}$ (for $i=j_{1}, j_{2}, \cdots, j_{v}$ ) in the access instance $f_{j}$ can cooperate to decrypt the message by using their secret keys as follows:

Step 1. Compute the point $T_{i}=x_{i} \cdot B$, for $i=j_{1}, j_{2}, \cdots, j_{v}$.

Step 2. Compute $P_{M}=C_{j}-\left(\sum_{U_{i} \in f_{j}} T_{i}\right)$ and recover $M$ from the $x$-coordinate of $P_{M}$.

The correctness of the elliptic curve version of our proposed GGOC is described as follows:

Theorem 2. The users $U_{i}$ s (for $i=j_{1}, j_{2}, \cdots, j_{v}$ ) in the access instance $f_{j}$ can cooperate to compute $P_{M}$ and then recover the message $M$.

Proof. Because

$$
\begin{aligned}
C_{j} & =P_{M}+\left(\sum_{U_{i} \in f_{j}} Y_{i}\right) \cdot r \\
& =P_{M}+\left(\sum_{U_{i} \in f_{j}} x_{i} \cdot G\right) \cdot r,
\end{aligned}
$$

thus

$$
\begin{aligned}
P_{M} & =C_{j}-\left(\sum_{U_{i} \in f_{j}} x_{i} \cdot G\right) \cdot r \\
& =C_{j}-\left(\sum_{U_{i} \in f_{j}} x_{i} \cdot B\right), \\
& =C_{j}-\left(\sum_{U_{i} \in f_{j}} T_{i}\right) .
\end{aligned}
$$

For the same reason, if the access structure has only one single user, the function of this scheme is exactly the same as that of the elliptic curve ElGamal cryptosystem. The security of the elliptic curve version of GGOC, which is the same as that of the elliptic curve ElGamal cryptosystem, is based on the elliptic curve discrete logarithm problem (ECDLP). 


\section{Performances and Comparisons}

In this section, we shall compare the computational complexity performance of our schemes with that of Tsai et al.'s scheme. To analyze the computational complexity, we first define the following notations.

$T_{E X P}$ : the time for computing modular exponentiation.

$T_{M U L}$ : the time for computing modular multiplication.

$T_{I N V}$ : the time for computing modular inverse.

$T_{X O R}$ : the time for computing eXclusive-OR operation.

$T_{E C \_M U L}$ : the time for computing the multiplication of a number and a point on the elliptic curve.

$T_{E C \_A D D} / T_{E C_{-} S U B}$ : the time for computing the addition/subtraction of two points on the elliptic curve.

$T_{E_{K e y}(M)} / T_{D_{K e y}(C)}$ : the time for encrypting/decrypting the message $M / C$ by using the symmetric cryptosystem.

$n$ : the number of different users in the access structure.

$m: m=\sum_{i=1}^{n}\left(\#\left(U_{i}\right)-1\right)$, where \# $\left(U_{i}\right)$ denotes the number of access instances containing $U_{i}$.

$k$ : the number of access instances.

$v$ : the number of users in the access instance $f_{j}$.

In our GGOC with the ElGamal cryptosystem, to encrypt the message $M$, the sender $U_{0}$ computes $b$ in Step 1, which requires $1 \times T_{E X P}$. Then, in Step 2, the ciphertexts $C_{i} \mathrm{~s}$ (for $i=1,2, \cdots, k$ ) are computed, which requires $k \times T_{E X P}+(m+1) \times T_{M U L}$. The total computational complexity for encrypting the message is therefore $(k+1) \times T_{E X P}+$ $(m+1) \times T_{M U L}$.

After receiving $\left\{F, b, C_{1}, C_{2}, \cdots, C_{k}\right\}$ sent by $U_{0}, U_{i}$ s (for $i=j_{1}, j_{2}, \cdots, j_{v}$ ) in the access instance $f_{j}$ as a whole computes $t_{i}$ s in Step 1 , which requires $v \times T_{E X P}$. Then, in Step 2, they can cooperate to recover the message $M$, which requires $v \times T_{M U L}+1 \times$ $T_{I N V}$. The total computational complexity for decrypting the message is $v \times T_{E X P}+$ $v \times T_{M U L}+1 \times T_{I N V}$.

Like we have just seen in the performance analysis of the GGOC with the ElGamal cryptosystem, the sender's total computations and $f_{j}$ 's computations in the elliptic curve version of our proposed GGOC are $(k+1) \times T_{E C_{-} M U L}+(m+1) \times T_{E C_{-} A D D}$ and $v \times T_{E C \_M U L}+v \times T_{E C \_A D D}+1 \times T_{E C \_S U B}$, respectively.

The computational complexity of Tsai et al.'s scheme has been shown in (Tsai et al., 1999). Since $T_{E X P}$ is much larger than $T_{M U L}, T_{X O R}$ and $T_{I N V}$, we only compare the number of $T_{E X P}$ in the following descriptions. According to Table 1, the numbers of $T_{E X P}$ in $f_{j}$ 's computations of both Tsai et al.'s scheme and our scheme with the ElGamal cryptosystem increase by $v$. Due to the sender's computations, the numbers of $T_{E X P}$ in Tsai et al.'s scheme and our scheme with the ElGamal cryptosystem are increased by $n$ and $k$, respectively. Because $n$ and $k$ are different variables, we will have to discuss what scheme is suitable for real-world applications.

On the other hand, the authors of (Koblitz et al., 2000; Schroeppel et al., 1995; Win et al., 1996) have pointed out that the base point $G$ with order $p$ is a 160-bit prime in the 
Table 1

Computational complexities of Tsai et al.'s scheme and our schemes

\begin{tabular}{|c|c|c|}
\hline & Sender's computations & $f_{j}$ 's computations \\
\hline Tsai et al.'s scheme & $\begin{array}{l}(n+1) \times T_{E X P}+m \times \\
T_{M U L}+k \times T_{X O R}+1 \\
\times T_{E_{K e y}}(M)\end{array}$ & $\begin{array}{l}v \times T_{E X P}+(v-1) \times \\
T_{M U L}+k \times T_{X O R}+ \\
1 \times T_{D_{K e y}}(C)\end{array}$ \\
\hline $\begin{array}{l}\text { Our scheme using the } \\
\text { ElGamal cryptosystem }\end{array}$ & $\begin{array}{l}(k+1) \times T_{E X P}+(m+ \\
1) \times T_{M U L}\end{array}$ & $\begin{array}{l}v \times T_{E X P}+v \times T_{M U L} \\
+1 \times T_{I N V}\end{array}$ \\
\hline $\begin{array}{l}\text { The elliptic curve version } \\
\text { our proposed scheme }\end{array}$ & $\begin{array}{l}(k+1) \times T_{E C_{-} M U L}+( \\
m+1) \times T_{E C_{-} A D D}\end{array}$ & $\begin{array}{l}v \times T_{E C_{-} M U L}+v \times \\
T_{E C_{-} A D D}+1 \times T_{E C_{\_} S U B}\end{array}$ \\
\hline
\end{tabular}

elliptic curve ElGamal cryptosystem that offers approximately the same level of security

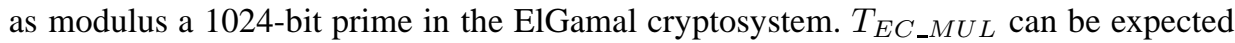
to be about 8 times faster than $T_{E X P}\left(8 \times T_{E C_{-} M U L}=T_{E X P}\right)$. Hence, the sender's computations and $f_{j}$ 's computations in the elliptic curve version are much more efficient.

\section{Discussions}

In this section, we shall consider a practical application of the GGOC and discuss the three scenarios ( $n>k, n=k$ and $n<k$ ). For example, suppose company has $N$ employees and $k$ departments. Here, we can take the company as the receiver group, the departments as the access instances $f_{1}, f_{2}, \cdots, f_{k}$, and the employees as the users $U_{1}, U_{2}, \cdots, U_{N}$.

Case 1: $n>k$

Assume that each department has more than one director. A managing director wants to send the message $M$ to the directors of all the departments such as the personnel department, administrative department etc., and only the directors in the same department can cooperate to decrypt this message. Here, $n$ is the number of directors whom the managing director sends the message to. In this case, no matter how many employees in the same department can cooperate to decrypt the message, $n$ is always greater than $k$.

Case 2: $n=k$

A managing director wants to send the urgent message $M$ to some specific employees in the company. Each of these employees can decrypt the message independently. The access structure can be presented as $F=U_{1}+U_{2}+\cdots+U_{n}$. For the same reason, if a managing director wants to send the urgent message $M$ to all the employees in the company, the access structure can be presented as $F=U_{1}+U_{2}+\cdots+U_{N}$. Here, $n$ is equal to $k$. 
Case 3: $n<k$

If the same employee belongs to more than one department at the same time, $n$ is less than $k$. Obviously, this rarely occurs. However, this situation occurs when GGOC extends to the threshold cryptosystem. In threshold cryptosystems such as (Desmedt and Frankel, 1989; Frankel, 1989; Hwang, 1990), the authorized subsets are all subsets of $t$ or more members of this group. The access structure with the threshold value $t$ can be represented as $F=U_{1} U_{2} \cdots U_{t}+U_{1} U_{2} \cdots U_{t-1} U_{t+1}+\cdots+U_{N-t+1} U_{N-t+2} \cdots U_{N}$. The number of access instances is $k=N ! / t !(N-t) !=n ! / t !(n-t) !$. However, when the GGOC schemes extend to be threshold cryptosystems, they become inefficient. The sender only encrypts the message by using the group's public key in the threshold cryptosystem.

The sender's computations in our scheme using ElGamal cryptosystem are more efficient than that in Tsai et al.'s scheme. The elliptic curve version of GGOC can further reduce the computations the sender and $f_{j}$ have to do. Furthermore, Tsai et al.'s scheme requires an additional symmetric cryptosystem to encrypt/decrypt the message, but ours do not.

\section{Conclusions}

Several GGOCs have been proposed previously (Chang and Lee, 1992; Chang and Lee, 1993; Lin and Chang, 1994). Among those GGOC-related schemes, Tsai et al.'s scheme (Tsai et al., 1999), reviewed in this paper, is one of the most efficient. In this article, we have shown that the computational complexity of our scheme using the ElGamal cryptosystem is lower than that of Tsai et al.'s scheme. Besides, our elliptic curve version of GGOC is much more efficient in the sender's computations and $f_{j}$ 's computations. Furthermore, Tsai et al.'s scheme requires an additional symmetric cryptosystem to encrypt/decrypt the message, while ours do not.

\section{Acknowledgements}

The authors wish to thank many anonymous referees for their suggestions to improve this paper. Part of this research was supported by the National Science Council, Taiwan, R.O.C., under contract no. NSC90-2213-E-324-004.

\section{References}

Chang, C.C., and H.C. Lee (1992). A solution to generalized group oriented cryptography. In IFIP/Sec'92Singapore Day2/Track2-Cryptography. pp. 289-299.

Chang, C.C., and H.C. Lee (1993). A new generalized group-oriented cryptoscheme without trusted centers. IEEE Journal on Selected Area in Communication, 11(5), 725-729.

Daemen, J., and V. Rijmen (1999). AES Proposal: Rijndael. Tech. Rep., Document Version 2.

Daemen, J., and V. Rijmen (2001). Rijndael, the advanced encryption standard. Dr. Dobb's Journal, 26(3), 137-139. 
Desmedt, Y. (1987). Society and group oriented cryptography: A new concept. In Advances in Cryptology, CRYPTO'87. pp. 120-127.

Desmedt, Y., and Y. Frankel (1989). Threshold cryptosystem. In Advances in Cryptology, CRYPTO'89. pp. 307315.

Diffie, W., and M. Hellman (1976). New direction in cryptography. IEEE Transactions on Information Theory, 22(6), 472-492.

ElGamal, T. (1985). A public-key cryptosystem and a signature scheme based on discrete logarithms. In IEEE Transactions on Information Theory, IT-31. pp. 469-472.

Frankel, Y. (1989). A practical protocol for large group oriented networks. In Advances in Cryptology, CRYPTO'89. pp. 56-61.

Hwang, M.-Sh., Ch.-Ch. Chang and K.-F. Hwang (2002). An ElGamal-like cryptosystem for enciphering large messages. In IEEE Transactions on Knowledge and Data Engineering, 14(2). pp. 445-446.

Hwang, T. (1990). Cryptosystem for group oriented cryptography. In Advances in Cryptology, EUROCRYPT'90. pp. 317-324.

Koblitz, N. (1987). Elliptic curve cryptosystems. Mathematics of Computation, 48, 203-209.

Koblitz, N. (1998). Algebraic aspects of cryptography. Algorithms and Computation in Mathematics, 3.

Koblitz, N., A. Menezes and Scott A. Vanstone (2000). The state of elliptic curve cryptography. Designs, Codes and Cryptography, 9(2/3), 173-193.

Lin, C.H., and C.C. Chang (1994). Method for constructing a group-oriented cipher system. Computer Communications, 17(11), 805-808.

Miller, V. (1986). Use of elliptic curves in cryptography. In Advances in Cryptology, CRYPTO'85. pp. 417-426.

Schroeppel, R., H. Orman, S. O’Malley and O. Spatscheck (1995). Fast key exchang with elliptic curve systems In Advances in Cryptology, CRYPTO'95. pp. 43-56.

Smid, M.E., and D. K. Branstad (1988). The data encryption standard: Past and future. In Proc. of the IEEE, 76. pp. 550-559.

Stallings, W. (1999).it Cryptography and Network Security: Principles and Practice. Prentice Hall, second edition.

Tsai, J.J., T. Hwang and C.H. Wang (1999). New generalized group-oriented cryptosystem based on DiffieHellman scheme. Computer Communications, 22(8), 727-729.

Win, E. De, A. Bosselaers, S. Vandenberghe, P. De Gersem and J. Vandewalle (1996). A fast software implementation for arithmetic operations in $G F\left(2^{n}\right)$. In Advances in Cryptology, Asiacrypt'96. pp. 65-76.

C.-C. Yang received his B.S. in Industrial Education from the National Kaohsiung Normal University, in 1980, and his M.S. in Electronic Technology from the Pittsburg State University, in 1986, and his Ph.D. in Computer Science from the University of North Texas, in 1994. He has been an associate professor in the Dept. of Computer Science and Information Engineering since 1994. His current research interests include network security, mobile computing, and distributed system.

T.-Y. Chang received the B.S. in Information Management from Chaoyang University of Technology (CYUT), Taichung, Taiwan, Republic of China, in 1999 and 2001. He is currently pursuing his master degree in Computer Science and Information Engineering from CYUT. His current research interests include information security, cryptography, and mobile communications.

J.-W. Li received the B.S. in Information Engineering and Computer Science from Feng Chia University, Taichung, Taiwan, Republic of China, from 1997-2001. He is currently pursuing his M.S. in Computer Science and Information Engineering from CYUT. His current research interests include information security, cryptography, and mobile communications. 
M.-S. Hwang received the B.S. in Electronic Engineering from National Taipei Institute of Technology, Taipei, Taiwan, Republic of China, in 1980; the M.S. in Industrial Engineering from National Tsing Hua University, Taiwan, in 1988; and the Ph.D. in Computer and Information Science from National Chiao Tung University, Taiwan, in 1995. He also studied Applied Mathematics at National Cheng Kung University, Taiwan, from 1984 1986. Dr. Hwang passed the National Higher Examination in field "Electronic Engineer" in 1988. He also passed the National Telecommunication Special Examination in field "Information Engineering", qualified as advanced technician the first class in 1990. From 1988 to 1991, he was the leader of the Computer Center at Telecommunication Laboratories (TL), Ministry of Transportation and Communications, ROC. He was also a project leader for research in computer security at TL in July 1990. He obtained the 1997, 1998, and 1999 Distinguished Research Awards of the National Science Council of the Republic of China. He is currently a professor and chairman of the Department of Information Management, Chaoyang University of Technology, Taiwan, ROC. He is a member of IEEE, ACM, and Chinese Information Security Association. His current research interests include database and data security, cryptography, image compression, and mobile communications.

\title{
Nesudètingas, grupei skirtas, apibendrintas šifravimas, naudojant EIGamal šifravimo sistema
}

\author{
Chou-Chen YANG, Ting-Yi CHANG, Jian-Wei LI, Min-Shiang HWANG
}

Apibendrintoje, grupei skirtoje šifravimo sistemoje vartotojas gali siųsti sąlygini pranešima vartotoju grupei taip, kad tik išskirtiniai vartotoju pogrupiai, iššifruodami tą žinutę, gali bendrauti grupeje. Tam, kad būtụ galima pasiekti apibendrinimo ir grupinio bendravimo tikslus, šiame straipsnyje naudojamos dvi šifravimo sistemos: ElGamal šifravimas ir ElGamal elipsinès kreivès šifravimas. Abi pasiūlytos sistemos yra efektyvesnès už Tsai et al. schemą skaičiavimo sudètingumo siuntejo pusejje atžvilgiu. 